\title{
Yoga as a Treatment for Low Back Pain: A Review of the Literature
}

\author{
Erik J. Groessl1,2, Marisa Sklar ${ }^{3}$ and Douglas Chang ${ }^{1,2}$ \\ ${ }^{1} V A$ San Diego Healthcare System, \\ ${ }^{2}$ University of California San Diego, \\ ${ }^{3} S D S U / U C S D$ Joint Doctoral Program in Clinical Psychology, \\ USA
}

\section{Introduction}

Chronic low back pain (CLBP) affects millions of people worldwide. In addition to chronic pain, CLBP is associated with increased disability and psychological symptoms, and reduced health-related quality of life (HRQOL). There are many treatment options for chronic low back pain, although no single therapy stands out as being the most effective. In the past 10 years, yoga interventions have been studied as an additional approach for treating CLBP. The objective of this chapter is to provide an introduction to yoga as a treatment for CLBP before reviewing the published literature to date supporting the efficacy of yoga for CLBP. Two large randomized controlled trials (RCTs) published late in 2011 provide the most conclusive evidence to date in this area. With few exceptions, previous studies and the recent RCTs indicate that yoga can reduce pain and disability, can be practiced safely, and is well received by participants. Some studies also indicate that yoga can reduce pain medication use and improve psychological symptoms, but these effects are currently not as well established. We summarize these results, discuss their implications, and examine caveats and limitations of the current research evidence. Finally, we provide suggestions for future avenues of research.

\section{Chronic low back pain}

Back pain is the second most common reason for physician visits and approximately $25 \%$ of the US population report having had back pain that lasted all day in the prior 3 months. (Deyo, Mirza, \& Martin, 2006) It is estimated that $90 \%$ of all acute back pain episodes resolve within 4 weeks, (Anderson, 1997) up to a third of those who sought treatment for their back pain reported persistent pain one year later, (Von Korff \& Saunders, 1996) with 20\% also reporting limitations of activity. It was estimated that back pain-related health care costs were about $\$ 26$ billion in 1998 and that the health care costs of back pain patients are $60 \%$ higher than those without back pain. (Luo, Pietrobon, Sun, Liu, \& Hey, 2004)

Low back pain is the most common type of back pain and is a prevalent condition that afflicts about $70 \%$ of people in developing countries at some point in their lifetime. (Anderson, 1997) The incidence of low back pain is greatest in persons of young adult and 
middle age, with $74 \%$ of all health visits for low back pain made by persons between the ages of 18 and 64 years. (AAOS, 2008) Additionally, in this group, low back pain is often associated by reduced ability to work or inability to work at all. The total economic impact (health care costs plus socioeconomic costs) has therefore been estimated at over $\$ 100$ billion each year. (AAOS, 2008) In the past, chronic low back pain was defined as low back pain lasting 12 weeks or more, but current guidelines now primarily differentiate between acute low back pain lasting less than 4 weeks and chronic, subacute low back pain lasting greater than 4 weeks. (Chou et al., 2007) This distinction coincides with the data cited above showing most back pain resolves on its own within 4 weeks.

Chronic low back pain results in more than just pain and discomfort. Persons with chronic low back pain also experience a variety of other symptoms and functional limitations. These include increased psychological symptoms such as depression, (Currie \& Wang, 2004; Sullivan, Reesor, Mikail, \& Fisher, 1992) anxiety, (Manchikanti, Pampati, Beyer, Damron, \& Barnhill, 2002; Thompson, Bower, \& Tyrer, 2007) increased disability, (Guo, Tanaka, Halperin, \& Cameron, 1999) and reduced health-related quality of life (HRQOL). (Burstrom, Johannesson, \& Diderichsen, 2001; Kosinski et al., 2005) Low back pain has a major impact on workforce productivity, with millions of workdays (Guo et al., 1999) (costing billions of dollars) lost for employees with back pain each year in the US. Research on disability and lost productivity among people with low back pain indicates that psychological factors are independently associated with lost productivity, increased disability, (Schiphorst Preuper et al., 2007) and increased healthcare utilization. (Keeley et al., 2008) Using formal diagnostic criteria, results of a 2004 research study found that $20 \%$ of persons with chronic back pain had major depression while only $6 \%$ of pain-free individuals were classified this way. (Currie \& Wang, 2004) Higher rates of depression among individuals with low back pain have been found previously in many other studies, (Manchikanti et al., 2002; Sullivan et al., 1992) along with elevated rates of other disorders such as anxiety and somatoform disorder. (Manchikanti et al., 2002) Although the causal relationship between psychological symptoms and CLBP is complex, research evidence indicates that psychological symptoms often improve in low back pain patients after exercise interventions, even if the interventions were not specifically designed to affect the psychological symptoms. (Roche et al., 2007) Conversely, placebo or sham treatments for low back pain have not resulted in significant changes in psychological symptoms. (Thompson et al., 2007)

\section{Current treatments for chronic low back pain}

Treatment recommendations for low back pain have been updated in 2007 and begin with an effort to categorize patients into three groups: Nonspecific low back pain, low back pain with radiating leg pain, and low back pain from a specific cause (e.g. fracture, malignancy, infection, cauda equine syndrome, or ankylosing spondylitis). (Chou et al., 2007) The majority of chronic low back pain cases (85\%) are nonspecific and are not linked to specific physical abnormalities. (van Tulder, Assendelft, Koes, \& Bouter, 1997) When a treating physician deems low back pain to be non-specific, health care providers are strongly recommended to begin treatment by providing patients with evidence-based information and to also encourage them to stay active and perform self-care activities. Self-care or selfmanagement activities are strongly recommended because they are inexpensive and are almost as effective as other non-pharmacological options. Another front-line option for patients with nonspecific chronic back pain is medication. (Chou \& Huffman, 2007a; Chou et 
al., 2007) Patients that do not improve after treatment with self-care activities and/or medications are good candidates for non-pharmacological treatments. Non-pharmacological treatments include physical treatments (i.e. heat, ice, ultrasound, massage therapy), spinal manipulation, and forms of injection therapy. (Chou \& Huffman, 2007b) Other studies have examined interventions such as exercise therapy, yoga, back schools, acupuncture, psychological therapies, laser treatment, lumbar supports, traction, and transcutaneous electrical nerve stimulation.

\section{Non-pharmacological treatments}

Non-pharmacological treatments are diverse, and vary considerably in the quality and amount of evidence supporting them and in the effect sizes they produce ${ }^{14}$. Despite varying evidence of their effectiveness, non-pharmacologic treatment options are widely recommended, especially by primary care physicians when their patients have not improved. (Di Iorio, Henley, \& Doughty, 2000; Freburger, Carey, \& Holmes, 2005) Ratings of the level of evidence for non-pharmacological treatments in recent guidelines are as follows: "good" scientific evidence was available for spinal manipulation (moderate effects), multidisciplinary approaches (moderate effects), exercise (small to moderate effects), and some psychological interventions (moderate effects), exercise (small to moderate effects), and some psychological interventions (moderate effects); (Chou \& Huffman, 2007b) a "fair" level of evidence was found for yoga (moderate effects), acupuncture (moderate effects), functional restoration (moderate effects), back schools (small effects), and continuous traction (not effective); and finally, effects were not rated for the seven other therapies for which evidence was judged as "Poor". Overall, the recommendations suggest that several non-pharmacological treatments are moderately effective, but none stand out as exceptionally beneficial. Thus, the characteristics, preferences and resources of individual patients, the cost and risk of the interventions, and consideration of the disease's natural history itself become important factors in treatment decision-making for chronic low back pain. For instance, some patients are at greater risk for developing addictions when treated with narcotic pain medications, making non-pharmacological treatments a more appealing choice.

\section{Medical procedures}

Injectional therapy to treat back conditions has become increasingly popular. Some injections (e.g. trigger point injections, acupuncture, prolotherapy) can be considered one of the many sundry non-pharmacological treatments. Other injections (e.g. epidural steroid injections, facet injections, medial branch blocks, radiofrequency ablation) are performed more frequently for spinal conditions and deserve specific discussion. The utilization rates for injectional therapies has risen about 250\% between 1994 and 2001. (Chou, Atlas, Stanos, \& Rosenquist, 2009) However, the evidence for these therapies is controversial. There are few well designed studies that support their usage and many studies with limitations that show either benefit or no benefit.

The best evidence supports the use of epidural steroid injections in the treatment of radicular pain. Bush et al. (Bush, Cowan, Katz, \& Gishen, 1992) followed 165 patients with lumbar radicular pain for one year. The patients were given $\sim 3$ lumbar epidural steroid injections (using a caudal approach). 14\% of the patients opted for surgical decompression, the rest had a satisfactory clinical recovery, with $94 \%$ reduction in visual analog scale pain and partial to complete resolution of disk herniations and disk bulges. Riew et al. followed 
55 patients for five years with lumbar radicular pain who had initially opted for surgery. (Riew et al., 2006) The patients were offered lumbar epidural steroid injection therapy (using a transforaminal approach) prior to the planned surgery. After two years, 29 of the patients avoided surgery. After five years, 21 of the 29 patients were identified and re-evaluated. Four of the 21 patients had opted for surgical treatment, while 17 continued to avoid surgery. These 17 had significantly decreased neurological symptoms and pain.

Facet joint injections and medial branch blocks have been proposed to treat axial (nonradiating) low back pain. While there is good research to support their usage in cervical spine pain, the evidence to treat lumbar pain is more limited. (Chou et al., 2009) In one study, 95 patients were followed for 6 months following a beneficial response to anesthetic injections into the lumbar facet joints of subjects with back pain. (Carette et al., 1991) The patients were randomized for another treatment with cortisone versus saline. Variable treatment responses were observed. There was no additional benefit of steroid over saline after the initial anesthetic injection.

Lastly, surgical treatment of non-specific low back pain has not been shown to be reliably successful. (Zigler et al., 2007) Depending on the outcome measure or surgery utilized, "success" rates for surgery for low back pain only range from $40 \%-65 \%$.

\section{Yoga}

The word "yoga" means union, and refers to the goal of uniting individual human spirit or will with divine spirit or the True Self. (Bhaktivedanta Swami Prabhupada, 1997; Stiles, 2000) Classical yoga (Raja Yoga) is an ancient discipline that was first formally described by Patanjali around $200 \mathrm{BC}$ in the Yoga Sutras. It has roots in Hindu religion and philosophy (Stiles, 2000) and was designed to create harmony of mind and body, and aid in achieving enlightenment or oneness with God. Although yoga has at times been misunderstood in the West as primarily "stretching", the postures or poses ('asanas'), comprise just one of eight components of a broader discipline of comprehensive physical, mental, and spiritual health and balance for individuals. However, many types of yoga do not include or do not emphasize stretching or postures, and are not considered Hatha yoga. (Sivananda, 1999; Yogananda, 1998) Modern Hatha yoga usually includes other classical yoga components such as breathing exercises ('pranayama'), concentration (pratyhara), and mindfulness/meditation (dhyana). (Iyengar, 1979) Thus, a typical Hatha yoga program consists of an instructor leading a group of students or practitioners through a series of yoga postures while performing deep breathing exercises for. Most classes last 60-90 minutes, and the instructor demonstrates the correct posture for practitioners, and provides verbal suggestions that encourage practitioners focus their attention or concentrate on deep breathing, postural alignment, on bodily sensations produced by the asanas. Depending on their training and often the class setting, instructors often encourage students to embrace positive cognitions or attitudes towards the world and themselves. This usually occurs at the beginning or end of the session and the instructors model these attitudes as well. Some yoga instructional centers provide a more complete social and spiritual community in which the additional components of classical yoga are also practiced.

There are many different types of Hatha yoga (Ashtanga, Anusara, Viniyoga, Bikram, etc). These styles or schools of Hatha yoga differ on variables such as pace, or the amount of time 
spent in each pose, the extent to which deep breathing is emphasized, level of emphasis on proper bodily alignment, room temperature, spiritual emphasis, meditation time, and the overall intensity and difficulty of the poses. Hundreds or possibly thousands of postures and variations have been developed over time, each one designed to stretch, strengthen, or engage specific areas of the body. When modified, Hatha yoga can be practiced by almost anyone, not just the healthy and flexible. This can be achieved by the use of props which enables people of all ages and ability levels (physical and mental) to perform poses that achieve benefit. (Iyengar, 1979) Yoga can be tailored to people with various physical or psychological limitations and can range from gentle to strenuous, with some types of yoga providing a cardiovascular workout, and others focused on relaxation and a calm mind. To be improve physical and mental health, the practice of yoga is usually performed at least once per week (twice is recommended), with increasing frequency of practice either at home or in a class, and gradual practice of more advanced postures as conditioning improves and strength increases. (Stiles, 2000)

The popularity of yoga has grown tremendously in recent years. Data from the National Center for Complementary and Alternative Medicine (NCCAM) show that the usage of complementary and alternative medicine (CAM) treatments for all conditions is on the rise in the US. Back pain is the most common condition for which CAM treatments are sought. Yoga was the $5^{\text {th }}$ most commonly used CAM treatment and its use increased significantly between 2002 and 2007. Yoga was used by $6.1 \%$ of all US adults and $2.1 \%$ of all children in the use in 2007.

\section{Broader effectiveness of yoga therapies}

Although there are many studies claiming yoga can be an effective treatment for improving a wide variety of conditions (musculoskeletal problems, (Greendale, McDivit, Carpenter, Seeger, \& Huang, 2002) cardiopulmonary function, (Raub, 2002) lipid and carbohydrate metabolism, (Bera \& Rajapurkar, 1993) sleep problems, (Harinath et al., 2004) anxiety and depression, (Waelde, Thompson, \& Gallagher-Thompson, 2004) ) study quality has been lacking, with a clear need for larger randomized, controlled trials (RCTs). (Luskin et al., 2000) The limitations of most previous yoga studies include being nonrandomized, a lack of validated outcome measures, a lack of data on dose response, and little examination of the underlying mechanisms. In addition, the yoga interventions being studied are often not well described. Yoga is multi-dimensional, so it is important to describe all of the components of each yoga intervention in order to compare across interventions and better understand which components are best for different disease populations. More recently, some larger, higher quality randomized studies have been conducted in areas including depression, (Sharma, Das, Mondal, Goswampi, \& Gandhi, 2005) stress and anxiety, (Granath, Ingvarsson, von Thiele, \& Lundberg, 2006; Smith, Hancock, Blake-Mortimer, \& Eckert, 2007) HIV, (Brazier, Mulkins, \& Verhoef, 2006) irritable bowel syndrome, (Kuttner et al., 2006) and chronic low back pain. (Sherman et al., 2011; Tilbrook et al., 2011) With very few exceptions, these more recent trials indicate that yoga has demonstrable beneficial effects, is rarely harmful, and is well received by participants with a wide variety of health problems. The psychological benefits of yoga are almost as well established as the physical benefits, as suggested by studies on stress, anxiety, and depression. 
In summary, chronic low back pain affects millions of people on a daily basis, and while there are many treatment options, none stand out as being highly effective. Yoga is a promising inexpensive alternative for treating CLBP with few anticipated side effects. It has moderate effectiveness and the next section reviews the published literature in more detail, including two large RCTs that were recently published.

\section{Literature review: Yoga for chronic low back pain}

\section{The impact of yoga on physical functioning and disability}

Almost all studies of yoga intervention for treating chronic low back pain measure physical functioning/disability as a primary outcome. It is viewed as a more reliable and objective measure of CLBP because it is often either measured by actual physiological performance, or by questionnaires with items that are tied to specific behaviors. Pain severity is typically an internal experience and more subjective. Virtually all studies measuring functioning/disability have demonstrated beneficial effects of yoga among adults with CLBP. (See Table 1) In 2004, Galantino et al. conducted a small randomized controlled trial to assess the impact of Hatha yoga on chronic low back pain. (Galantino et al., 2004) Participants were randomized to either the yoga group, consisting of twice-weekly, 60minute, yoga classes for 6 weeks, or a wait-list control. Outcomes related to physical functioning included flexibility as assessed with the forward reach and sit and reach tests, and disability as assessed with the Oswestry Disability Index. Results of this study demonstrated non-significant trends towards improved balance and flexibility and decreased disability for the yoga group. However, sample size and participant attrition weakened this study's power, demonstrating the need for larger studies assessing the impact of yoga on chronic low back pain. Additionally, the 6-week yoga program is shorter than the 10-12 week programs that are most frequently studied.

Williams et al. conducted a randomized controlled trial to assess the impact of Iyengar yoga therapy in participants with non-specific chronic low back pain. (K. A. Williams et al., 2005) The study compared a standardized yoga intervention to an educational control group. Both the intervention group and the control group programs were 16 weeks long. Both groups received 16 weekly newsletters on back care, and two lectures of occupational/physical therapy education regarding chronic low back pain with instructional handouts. The yoga intervention consisted of one 90-minute class each week for 16 weeks at a community yoga studio, and participants were encouraged to practice yoga at home for 30-min, 5 days a week. Study results revealed less functional disability in the yoga group than in the control group at the post-treatment assessment.

The efficacy of a week-long intensive residential yoga program on disability caused by pain and spinal flexibility in patients with chronic low back pain was studied by Tekur et al. (Tekur, Singphow, Nagendra, \& Raghuram, 2008) Eighty participants who were previously admitted to a health home in Bangalore, India were randomized to yoga and control groups. The yoga group followed a daily routine of meditation, yogic physical practices, yogic hymns, lectures on yogic lifestyle, yogic breathing, deep relaxation, counseling, and meditation with yogic chants. The control group followed a daily routine of exercise, nonyogic safe breathing exercises and lectures on causes of back pain, stress and chronic low back pain, and benefits of physical exercises. Control participants also watched video shows 


\begin{tabular}{|c|c|c|c|c|c|c|}
\hline 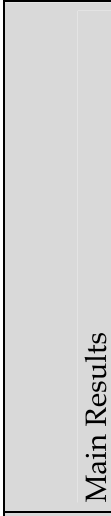 & 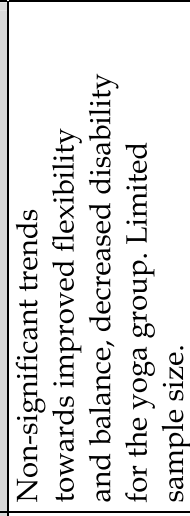 & 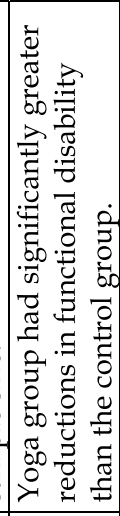 & 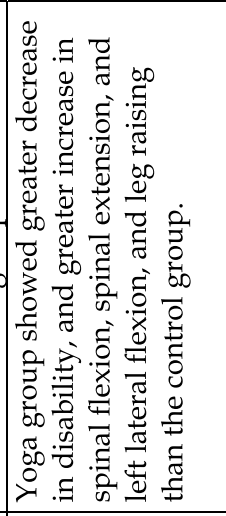 & 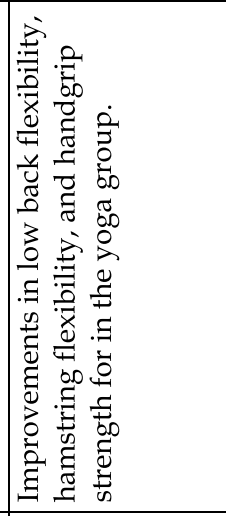 & 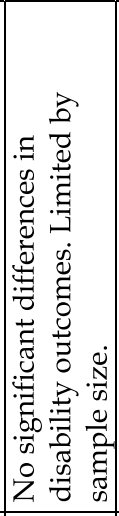 & 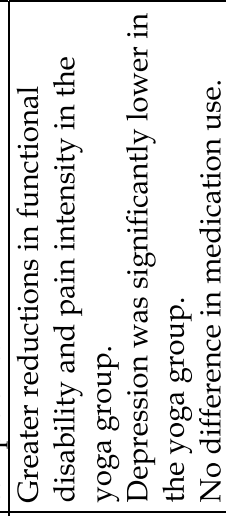 \\
\hline 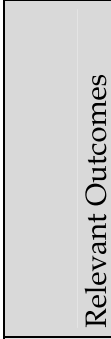 & 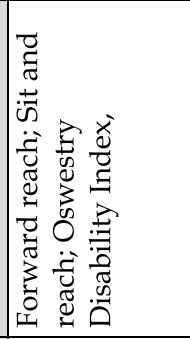 & 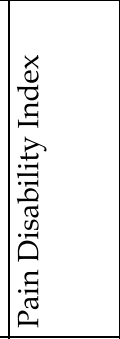 & 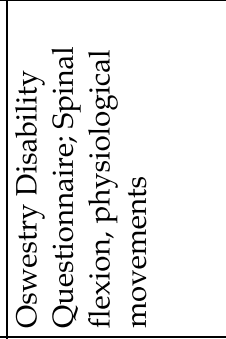 & 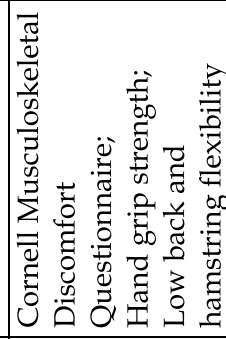 & 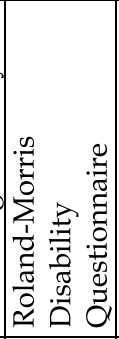 & 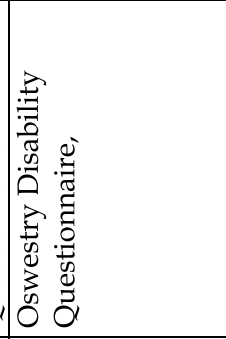 \\
\hline 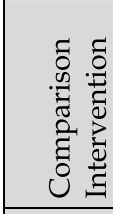 & 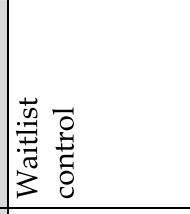 & 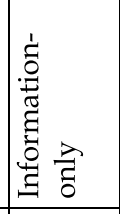 & 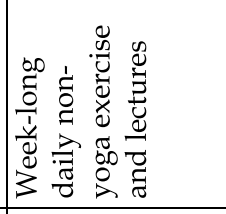 & 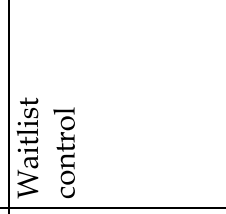 & 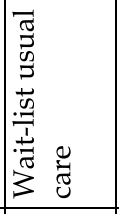 & 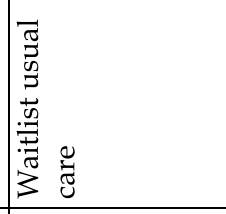 \\
\hline 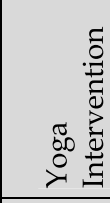 & 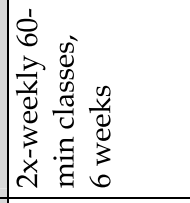 & 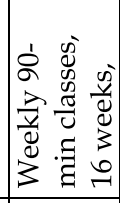 & 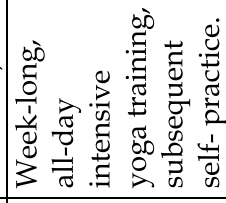 & 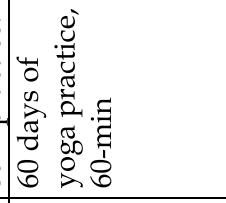 & 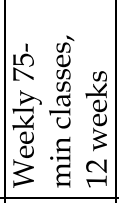 & 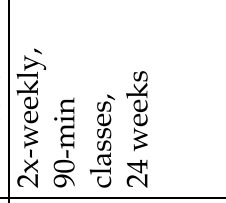 \\
\hline 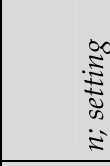 & $\approx$ & 8 & 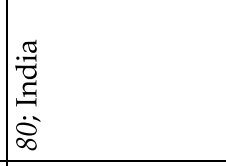 & 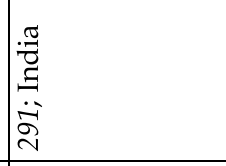 & 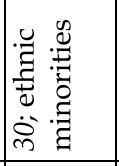 & ৪ \\
\hline 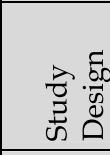 & 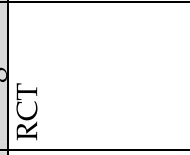 & 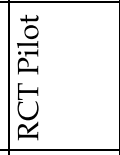 & 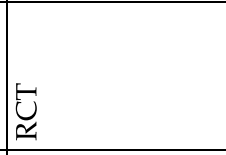 & 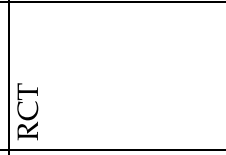 & 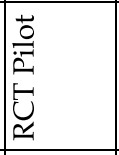 & 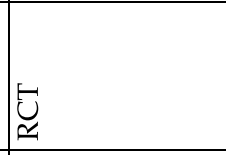 \\
\hline 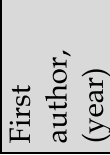 & $=\frac{0}{\stackrel{\Xi}{્}}$ & 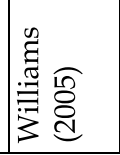 & 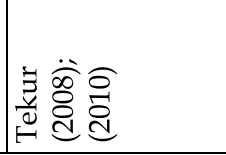 & 兽 & 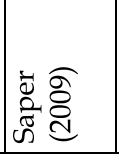 & 胥 \\
\hline
\end{tabular}

Table 1. Continued 


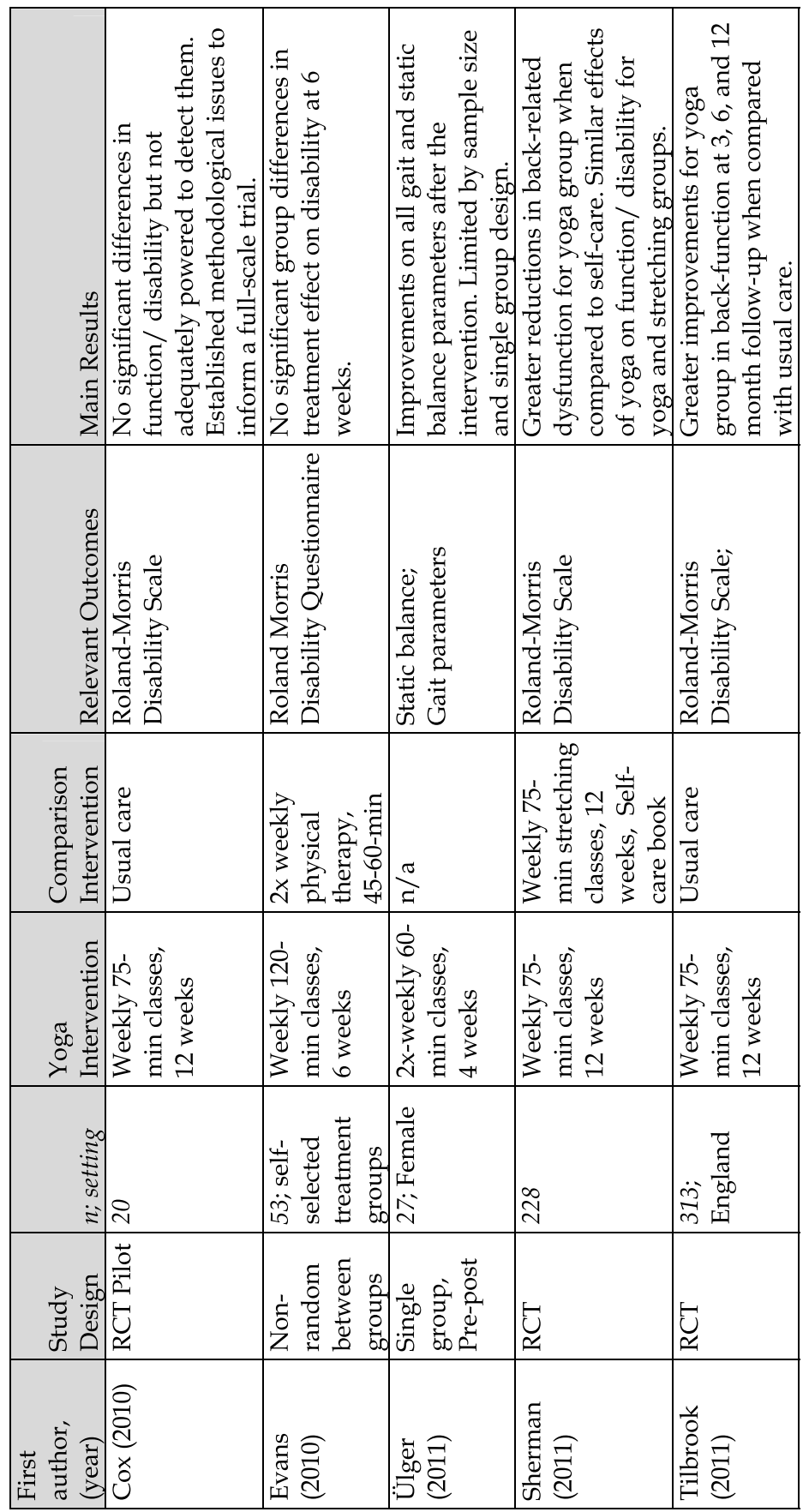

Table 1. Studies that examined the effect of yoga on functioning/disability. 
on animals, plants, nature, etc. Outcomes included spinal mobility, as measured using a dialtype goniometer, and the Oswestry Disability Index. Results showed a significant difference between groups in disability, with the yoga group experiencing a greater decrease in disability than the control group. Spinal flexion, spinal extension, and left lateral flexion, increased in both groups, with the yoga group showing a greater increase in flexibility than the control group. Greater improvements were also found in straight leg raises for right and left legs for yoga group. (Tekur, Chametcha, Hongasandra, \& Raghuram, 2010).

In 2009, Telles et al. presented results of a randomized control trial examining the effect of yoga on musculoskeletal discomfort and motor functions in professional computer users in India ( $\mathrm{n}=291$ ). (Telles, Dash, \& Naveen, 2009) Employees who used a computer for at least 6 hours each day, 5 days a week were randomized into a yoga intervention, or waitlist control. The yoga group participated in an hour of yoga practice each day, 5 days per week. Employees in the wait-list control spent the hour in a recreation center. Both groups were assessed at baseline and after 60 days on hand grip strength, tapping speed, and low back and hamstring flexibility. The yoga group demonstrated significant increases in handgrip strength for both hands and significant improvements in low back and hamstring flexibility. Results also demonstrated group differences in changes in tapping speed following the 60 days.

Williams et al. published additional results on the effectiveness of Iyengar yoga on chronic low back pain in 2009. (Williams et al., 2009) In this study, a total of 90 participants were randomized to the yoga intervention $(n=43)$ and wait-list control $(n=47)$ groups. The yoga intervention consisted of biweekly, 90-minute, Iyengar yoga classes over a 24 week period. Yoga participants were also asked to practice 30 minutes of yoga at home on non-class days, and were given props, a DVD, and an Iyengar yoga instruction manual. Participants in the control group continued their standard medical care, and were offered the yoga classes 6 months after the conclusion of the study. Individuals randomized to the yoga group showed greater improvements in functional disability than those randomized to the control group. Differences between the yoga and control groups were even stronger when limiting analyses to "completers" (30 individuals who completed the yoga intervention per protocol and 43 individuals not lost to follow-up in the waitlist control). Williams et al. (2009) report several limitations to their study, including reliance only on self-report measures, minimal disability among one-third of the participants, and the yoga group received greater attention and group support than did individuals from the control group.

In 2010, Cox et al. led a small randomized controlled pilot study $(n=20)$ with the goal of informing a larger multicenter trial on the effectiveness of yoga for chronic low back pain. (Cox et al., 2010) The study compared 12 weekly, 75-minute, yoga back classes with usual care. As part of the study, a yoga manual was developed for yoga practitioners and their students. Outcomes including the Roland-Morris Disability Scale were assessed at baseline, 4 and 12 weeks following randomization. No significant effects were found, as the pilot study was not powered to detect differences. The study did provide useful data for the larger study on feasibility of recruitment, attendance, and retention.

In 2010, Evans et al. studied the predictors of outcomes in individuals who self-selected yoga or physical therapy to treat chronic low back pain. (Evans et al., 2010) Specifically, yoga participants $(n=27)$ were recruited from a series of five 6-week, once weekly, yoga classes offered through a hospital based complementary and alternative medicine clinic. 
Physical therapy participants $(n=26)$ were recruited from 1 private and 1 hospital-based outpatient physical therapy clinic. Participants completed a clinical and demographic questionnaire at baseline and again after 6 weeks of treatment. Results suggested no significant group differences in treatment effect on disability at 6 weeks. However, their results indicated that self-efficacy was the most important predictor of disability and health status at 6 weeks for both groups. Additionally, the authors found a group by self-efficacy interaction upon predicting disability at 6 weeks. Specifically, self-efficacy was a stronger predictor of disability at 6 weeks for the physical therapy group. Because participants selfselected into the yoga or physical therapy group, a significant limitation of this study rests in fact self-selection bias may explain the differences in outcomes between the groups.

In 2011, Ülger and Yağli studied the effects of yoga on balance and gait in women $(n=27)$ with musculoskeletal disorders including low back pain and osteoarthritis. (Ülger and Yağli, 2011) The effect of 8 sessions of yoga treatment was assessed on gait (gait cycle, walking speed, maximum walking distance, step length, and ambulation index) and balance evaluations. Results suggested participants' gait parameters improved statistically following the 8 week yoga intervention. Additionally, improvements were also observed in the balance parameters. Although the authors conclude yoga has positive effects on physical problems such as gait and balance, significant limitations to this study include the absence of a control group/intervention, and small sample size. It is thus difficult to attribute the improvements in gait/balance to their yoga intervention.

Two larger randomized trials have recently been published in 2011 regarding yoga on chronic low back pain. Sherman et al. compared three different approaches designed to decrease the negative effects of back pain on participants' $(n=228)$ lives. (Sherman et al., 2011) Specifically, a series of yoga classes were compared to stretching exercises and to a self-care book. Yoga and stretching series consisted of twelve standardized, weekly 75-minute classes. Interviews were conducted at baseline and at 6, 12, and 26 weeks after randomization. Results suggested similar effects for yoga and stretching in individuals with low back pain. Back-related dysfunction declined over time in all groups, with the yoga group and stretching group reporting superior function than the self-care group at follow up assessments. At 12 weeks, the yoga group was significantly less bothered by symptoms than the self-care group. Both the yoga and stretching groups were more likely to rate their back pain as improved at all followup times, and were more likely to report being very satisfied with their care. Consequently, Sherman et al. suggest that yoga's benefits are largely attributable to the physical benefits of stretching and strengthening the muscles and not to its mental components.

In another study, Tillbrook et al. compared the effectiveness of yoga and usual care for chronic or recurrent low back pain using patients $(n=313)$ from 13 non-National Health Service premises in England, using long-term follow-up methods. (Tilbrook et al., 2011) The yoga intervention consisted of twelve 75-minute classes ( 1 class per week). Yoga participants were given a student manual, a mat, a relaxation compact disc which featured four narrated guided relaxations, and home practice sheets delivered at four intervals. Yoga participants were encouraged to use the compact disc, and to practice yoga for 30-minutes daily or to practice at least 2 times per week. All participants received a back pain education booklet and usual care. Additionally, fidelity assessments were used to ensure fidelity to the standardized treatment. Outcomes were measured before randomization, at baseline, and at 3, 6, and 12 months follow-up. Results suggested the 
yoga group had significantly greater improvements than the usual care group in backfunction at 3, 6, and 12 month follow-up. The authors conclude that a 12 week yoga intervention leads to greater improvements in back function than usual care treatment for up to 12 months.

\section{The impact of yoga on pain}

A number of studies, including many of the studies discussed above, also demonstrate the effectiveness of yoga in reducing pain in individuals with chronic low back pain. For example, in addition to studying yoga's effect on functional disability, Williams et al. (2005) also assessed clinical levels of pain, pain-related fears of movement, and pain attitudes. Their results suggested that in addition to the yoga group having less functional disability at post treatment, the yoga group demonstrated two times greater reductions in pain, than the control group.

Beginning in 2005, military veterans who began attending a clinical yoga program at a large VA medical center in California completed a battery of health questionnaires before and after attending a 10-week yoga program. Baseline data and 10-week follow-up data were available for 33 participants as of August 2007. Statistically significant improvements were found for pain between baseline and 10-weeks. Among the various indicators of the amount of yoga practiced, correlations indicated that actual attendance was significantly correlated with decreased pain. These effects were found despite the fact that some participants did not attend regularly and the sessions were only offered once per week. Participants were encouraged to practice yoga postures at home and self-reports of the frequency of home practice were also associated with improvements in back pain. Further analyses with an expanded sample $(n=53)$ from the same study indicate that women improved more than men in the yoga program after controlling for baseline differences. Female participants had significantly greater improvements than male participants for "average" pain levels. No differences were found between men and women for pain "at its worst" or a total pain score. Women and men did not differ on attendance or home practice.

Saper et al. (2009) also found greater decreases in pain scores for yoga participants than the control group from baseline to 12 weeks. The yoga group participants also reported larger global improvements in back pain at week 12 than control group participants. Results from this pilot study also provide support for the feasibility of recruiting, retaining, and treating a sample of predominantly minority adults for a 12 week yoga intervention. However, a number of limitations exist for this study. The small sample size limited their statistical power, there was substantial attrition to long-term follow-up in the yoga group, and many non-study treatments including yoga were used by the control group, making it difficult to draw conclusions from their 26-week data.

As discussed above, Telles et al. presented results of a randomized control trial examining the effect of yoga on musculoskeletal discomfort and motor functions in professional computer users $(n=291)$. (Telles, Dash, \& Naveen, 2009) At the end of 60 days, they found greater decreases in the degree of interference due to musculoskeletal discomfort in the yoga group. Limitations noted by the authors include a high attrition rate from the follow-up assessment in both groups, but attrition rates did not differ between the groups. Of the 57 individuals who dropped out from the yoga group, only 6 did so because they preferred to use the 60-minute period for a recreational activity of their choosing. A second limitation of 
the intervention they studied was the high level of commitment required to sustain a 5day/week, 60-minute, yoga practice on working days.

The 2009 study by Williams et al. discussed earlier also demonstrated the effectiveness of Iyengar yoga on improving pain for individuals with chronic low back pain. (K. Williams et al., 2009) Individuals randomized to the yoga group showed greater improvements in pain intensity than those randomized to the control group. Differences between the yoga and control groups were even stronger when limiting analyses to "completers" (30 individuals who completed the yoga intervention per protocol and 43 individuals not lost to follow-up in the waitlist control).

The Cox et al. study in 2010 measured pain-related outcomes using the Aberdeen Back Pain Scale and pain efficacy. At the 4-week follow-up, the yoga group reported greater decreases in pain. At both follow-up points, a non-significant trend in the yoga group showing an improvement in pain self-efficacy over the usual care group was observed. The results of the large RCT published by the same research group found no significant differences in pain severity at any assessment periods. They did find greater improvements in pain self-efficacy for the yoga group at $3 \& 6$ month follow-ups than those randomized to usual care.

In 2010, Evans et al. studied back pain bothersomeness, and back pain self-efficacy, in individuals who self-selected yoga or physical therapy to treat chronic low back pain. Results suggested no significant group differences in treatment effect on pain at 6 weeks. Their results also indicated that self-efficacy was the most important predictor of pain and health status at 6 weeks for both groups. Finally, Sherman et al. measured pain "bothersomeness" instead of pain severity because of the more subjective nature of reporting pain severity. Results indicate that the yoga group had significantlygreater reductions in pain "bothersomeness" than the self-care group at 12 weeks. However, they found similar effects of yoga on pain for yoga and stretching groups. Similar to the disability outcomes, both groups had reductions in pain after participating in the interventions.

\section{Medication use}

Five of the studies reviewed reported the effectiveness of yoga on reducing the use of medications. The results of Williams (2005) suggest a greater decrease in the use of pain medications than the control group. Saper et al. (2009) also reported on the beneficial effects of yoga on the use of medications. They found that the use of pain medicine differed significantly between the yoga and control groups such that the yoga participants decreased their use of pain medicine while the control group did not change. Opiate analgesic use increased for the control group participants, but decreased to zero for the yoga participants - another statistically significant difference between groups. Williams et al. (2009) found non-significant reductions in pain medication use at 12 and 24 weeks that was comparable in both their yoga group participants, and their control group who continued self-directed standard medical care. However, Williams et al. report a trend for the yoga group to have a higher success rate in decreasing their use of pain medication than at both 12 and 24 weeks follow up than the control group. Subgroup analyses that examined the use of pain medication in participants with moderate disability at baseline indicate that yoga participants with moderate disability showed a significantly greater reduction in pain medication at 12 weeks than their control group counterparts. In 2010, Evans et al. studied pain medication use in individuals who self-selected yoga or physical therapy to treat 
chronic low back pain. Results suggested that both self-selected groups decreased pain medication use by similar amount (52\% and 57\% among yoga physical therapy groups, respectively). In the largest study of the impact of yoga on medication use to date, Sherman et al. found greater decreases in medication use in the yoga group when compared to selfcare, but no difference between the yoga and stretching groups.

\section{Psychological impacts of yoga}

The broader effectiveness of yoga to the psychological well being of participants has been debated. Results from the literature are not yet conclusive regarding whether yoga can improve participants' psychological well being. For example, in 2004, Galantino et al. also assessed the impact of Hatha yoga on depression as assessed with the Beck Depression Inventory. Results of this study demonstrated non-significant trends towards decreased depression for the yoga group. However, sample size and participant attrition weakened this study's power, demonstrating the need for larger studies assessing the impact of yoga on chronic low back pain.

Groessl et al. also reported the influence of yoga on the psychological well being of participants among San Diego veterans. The study found significant improvements in depression as measured with the CES-D 10, and the Mental Health Scale of the SF-12. Among the various indicators of the amount of yoga practiced, correlations indicated that self-reported home practice was significantly correlated with improved outcomes for depression.

Williams et al. (2009) also reported on the impact of Iyengar yoga on the psychological well being of participants with chronic low back pain. Specifically, in reducing depression among individuals with chronic low back pain. Using the Beck Depression Inventory, individuals randomized to the yoga group showed greater improvements in depression than those randomized to the control group. As mentioned above, differences between the yoga and control groups were even stronger when limiting analyses to "completers".

In 2010, Tekur et al. (Tekur, Chametcha, Hongasandra, \& Raghuram, 2010) presented additional results from their 2008 study. The authors used the WHOQOL Bref psychological subscale to measure the impact of yoga on mental health. They found significantly greater improvements in WHOQOL Bref psychological subscale for the yoga group compared with the control group. However, the intervention included many more elements (formal meditation, interactive lectures, spirituality) than the typical Hatha yoga interventions being studied in the other research we have reviewed, and the benefits were documented only at 7 days after baseline.

Two studies by the same research group in the UK measured psychological impact with the Short From 12 Mental Component Scale (SF-12 MCS). The earlier pilot found no significant differences but has little power $(n=20)$ to detect differences. The Tilbrook study was adequately powered but found non-significant trends toward greater improvements among the yoga group at 3- and 6-month assessments.

\section{Other outcomes}

A variety of other outcomes were measured across the studies that have been reviewed. Some were very specific to the population being studied such as hand tapping speed for computer programmers. Other measures such as health-related quality of life were measured in a number of studies and are not study specific. 


\section{Safety of yoga}

It is important to address the concern that yoga could potentially be harmful to those with chronic low back pain. Popular media has tapped into this concern, often with anecdotal stories of dangers and injury, but little data. Like other exercise activity, the risks of injury from improperly performing yoga postures likely vary depending on how, where, and with whom the yoga is practiced. The initial practice of yoga under the direction of experienced yoga instructors is preferable to simply reading a book or following a video at home, and many of the programs being studied have been modified specifically for people with the condition of chronic low back pain. For optimal safety, people with either acute or chronic health conditions should consult their physician before starting a yoga program.

Data from research studies with experienced yoga instructors have shown occasional adverse events. The 2005 Sherman et al. study reported no serious adverse events. Of 36 patients assigned to the yoga group, one patient discontinued yoga because it precipitated migraines. Similarly, of 35 patients in the exercise group, one individual in the exercise group discontinued the intervention because of a back strain. Data from the Williams et al. study in 2005 show one serious adverse event among 30 patients randomized to yoga. This patient had symptomatic osteoarthritis and herniated a disc during the study. However, the event was reviewed by a medical panel and it was determined that the event was not caused by practicing yoga in this study. A larger, more recent study by Sherman et al. found equal numbers of mild to moderate adverse events in the yoga and stretching interventions, with temporary increases in back pain the leading cause. One serious adverse event, a herniated disc occurred among the 87 yoga participants. The other recent large RCT by Tilbrook et al. found 1 serious adverse event and 12 nonserious events among 156 yoga participants, all related to increased back pain. The more general review of nonpharmacological treatments for chronic low back pain concluded that these interventions seldom cause harm, but better studies and better reporting are needed (Chou \& Huffman, 2007b).

\section{Discussion}

Chronic low back pain is an extremely prevalent condition that results in a great deal of lost productivity, disability, discomfort, and reduced quality of life for those afflicted. CLBP patients have higher health care costs in both the short-term and long-term.

Current treatments for CLBP are variable in the quality of evidence supporting them and in their overall levels of effectiveness. Medication management works for some patients but others require stronger narcotic agents which heighten the risk of addiction. None of the non-pharmacologic treatments stand out as clearly superior. With the exception of exercise and yoga, most of the treatments with solid supporting evidence and at least moderately sized effects are performed in a one-on-one provider setting or require expensive equipment. Thus, treatment modalities such as spinal manipulation, physical therapy, acupuncture, etc. may be more expensive than yoga or exercise interventions that can be delivered in group format, or once learned, can be self-administered at home. Actual data and cost analyses on yoga interventions for chronic low back pain are needed.

Our review of the current literature indicates that yoga has reduced disability and improved daily functioning in most studies when compared with usual care, or information alone. Most studies use one of two well-validated measures of functioning/disability, and some studies also included physiological measures such as grip strength and flexibility (See Table 
1). The two most informative studies have been published in recent months and have not been included in previous reviews. Each of these recent studies found that yoga improved function more than usual care or self-care. The Sherman et al. study employed a three group design, and thus, provided important "comparative effectiveness" data by comparing yoga to an exercise program led by physical therapists. It is notable that was not statistically superior to this exercise intervention. The only other study that used a comparison group of proven efficacy was Evans et al who also found no significant differences on function/disability. Thus, we conclude that yoga may not be superior to other nonpharmacological interventions with moderate effects sizes. However, attendance and satisfaction rates were higher among the yoga group in the Sherman study, indicating that it may be a more attractive intervention to many individuals.

With the exception of a few smaller studies, yoga interventions have been shown to reduce pain severity or the "bothersomeness" of pain, when compared with usual care, or information alone (See Table 2). In looking at the recent RCTs, the Sherman study found that yoga patients were less bothered by low back pain than self-care patients, while the Tilbrook study found no significant differences in back pain severity. The Tilbrook finding is a bit surprising, even though self-reported pain severity is different than pain bothersomeness. Sherman et al. specifically chose to measure pain bothersomeness because self-reported pain severity may be harder to measure reliably across various groups. It is also possible that the design of the Tilbrook study (13 different private practices across the UK) may have affected the results obtained. This may have resulted in greater heterogeneity among the instructors, intervention, or participants, and it is unclear whether the statistical analysis accounted for clustering with the 13 cohorts.

Our review also suggests that yoga can reduce reliance on pain medication when compared with usual or self-care. However, only a few studies have published results on these outcomes so conclusions remain more tentative (See Table 3). With the exception of the Williams at el study, very little information is provided on how medication was measured. Measuring medication use poses its own challenges, with self-reported interview data often differing from medical record information or pill counts. Future research should consider other methods for measuring medication usage including the use of medical record information when possible.

Depression and other indicators of the impact of yoga on psychological outcomes were assessed in a small number of studies (See Table 4). Significant effects were found in a few of the smaller studies, but only non-significant trends were found for the SF-12 MCS in the fullscale trial in which they were measured. The Sherman study did not publish data on psychological variables in their initial manuscript but these results may be forthcoming. Given the cognitive and relaxation components of yoga, along with the higher rates of depression among individuals with chronic low back pain, further research in this area is very important.

We also reviewed the safety of yoga for some of the larger randomized controlled trials. Data from the largest and most recent trials suggests that two serious adverse events occurred among a combined total of 243 participants. These events were related to increased back pain, one being a herniated disc. Another $10 \%$ of these yoga participants experienced non-serious adverse events that were almost exclusively increases in back pain. Thus, participating in yoga interventions by persons with chronic low back pain is not without risk, but the vast majority of participants had no problems and experienced considerable benefit for a chronic debilitating condition. 


\begin{tabular}{|c|c|c|c|c|c|c|c|c|}
\hline 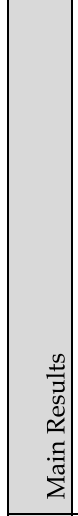 & 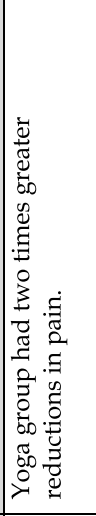 & 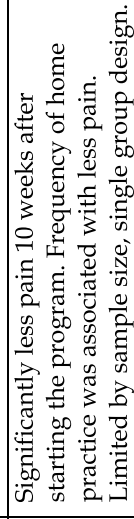 & 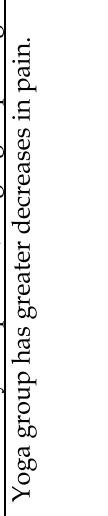 & 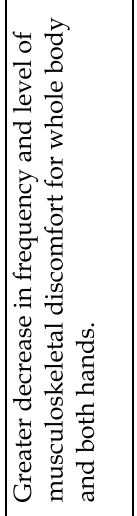 & 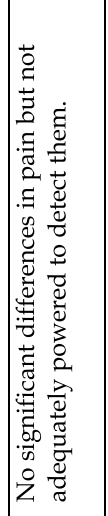 & 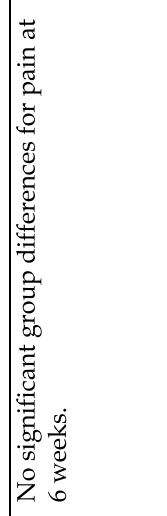 & 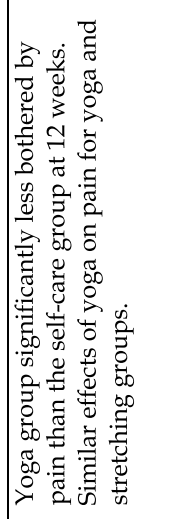 & 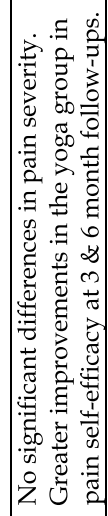 \\
\hline 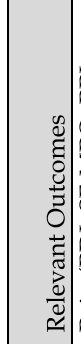 & 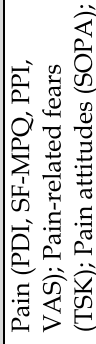 & 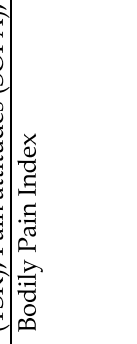 & 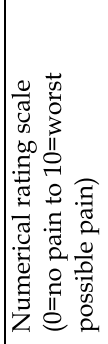 & 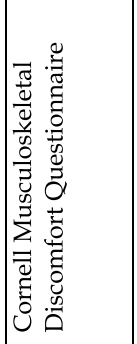 & 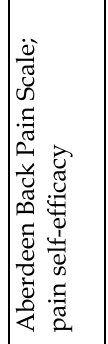 & 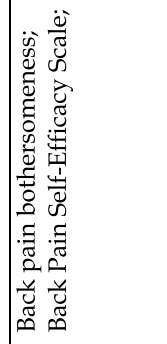 & 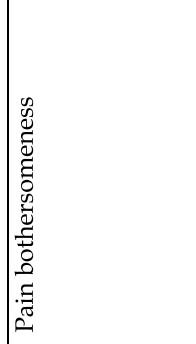 & 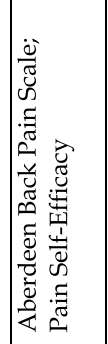 \\
\hline 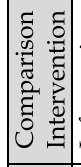 & 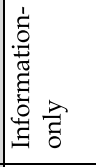 & $\stackrel{\sigma}{l}$ & 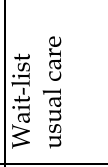 & 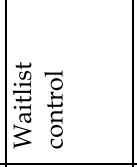 & 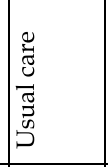 & 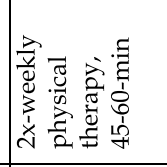 & 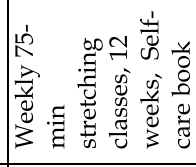 & 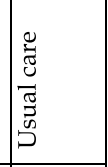 \\
\hline 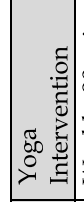 & 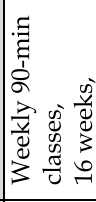 & 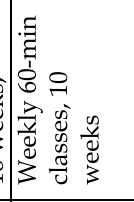 & 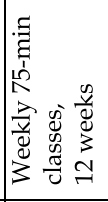 & 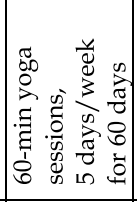 & 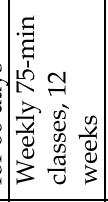 & 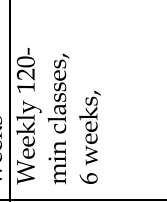 & 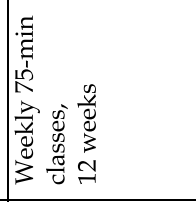 & 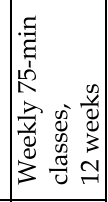 \\
\hline$=$ & 8 & 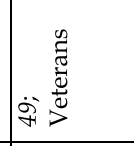 & 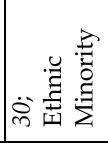 & 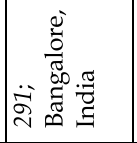 & సి & 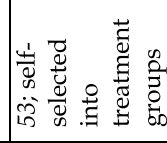 & $\mathbb{N}$ & 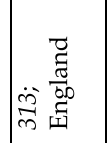 \\
\hline 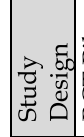 & 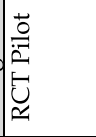 & 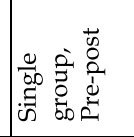 & 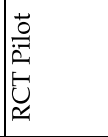 & $\approx$ & 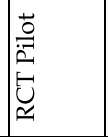 & 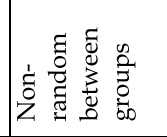 & 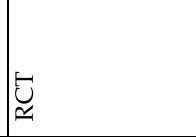 & $\overline{\mathscr{U}}$ \\
\hline 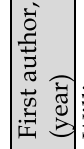 & 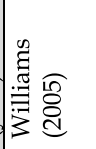 & 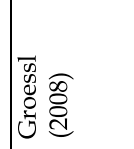 & 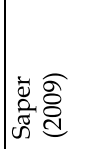 & 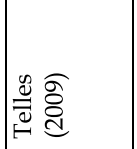 & 을 & 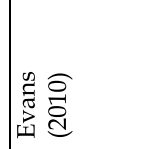 & 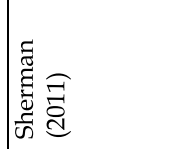 & 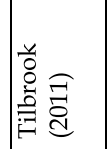 \\
\hline
\end{tabular}

Table 2. Studies that examined the effect of yoga on pain. 


\begin{tabular}{|c|c|c|c|c|c|}
\hline 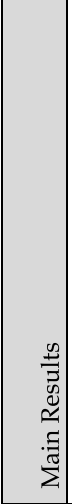 & 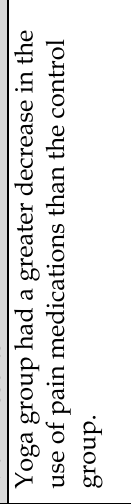 & 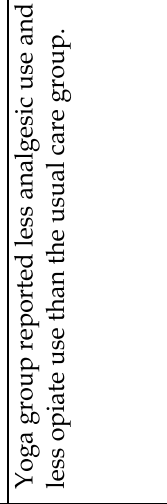 & 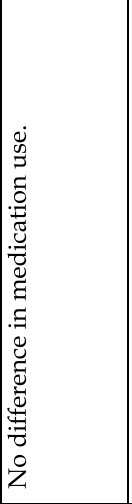 & 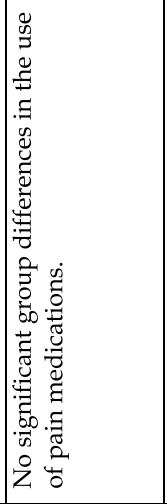 & 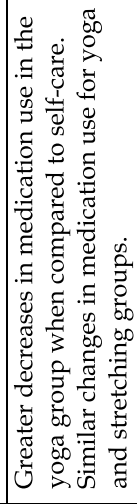 \\
\hline 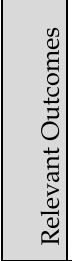 & 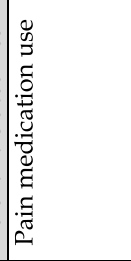 & 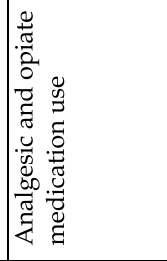 & 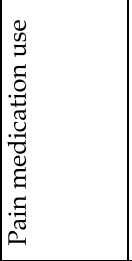 & 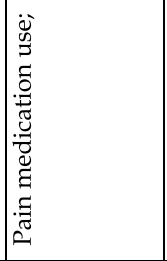 & 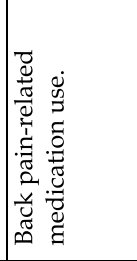 \\
\hline 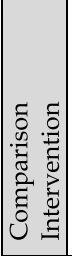 & 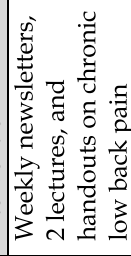 & 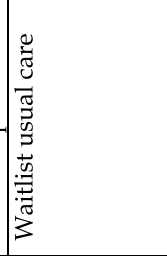 & 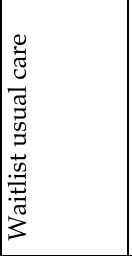 & 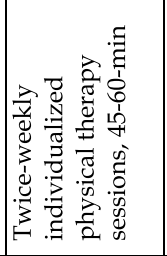 & 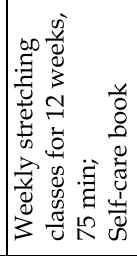 \\
\hline 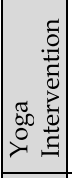 & 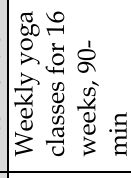 & 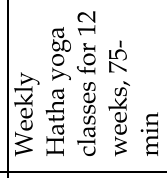 & 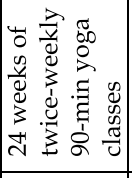 & 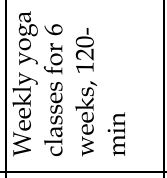 & 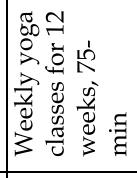 \\
\hline$=$ & 8 & 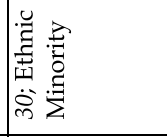 & 8 & 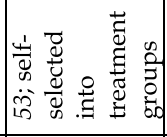 & \\
\hline 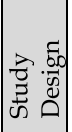 & 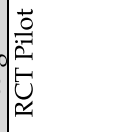 & 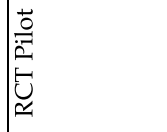 & $\underset{\simeq}{\tilde{\Xi}}$ & 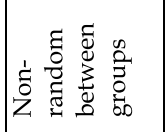 & $\widehat{\underline{U}}$ \\
\hline 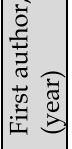 & 芶 & 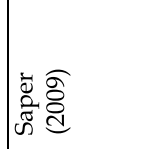 & 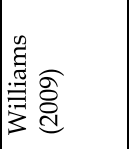 & 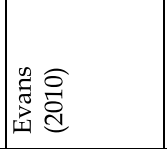 & 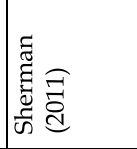 \\
\hline
\end{tabular}

Table 3. Studies that examined the effect of yoga on medication use. 


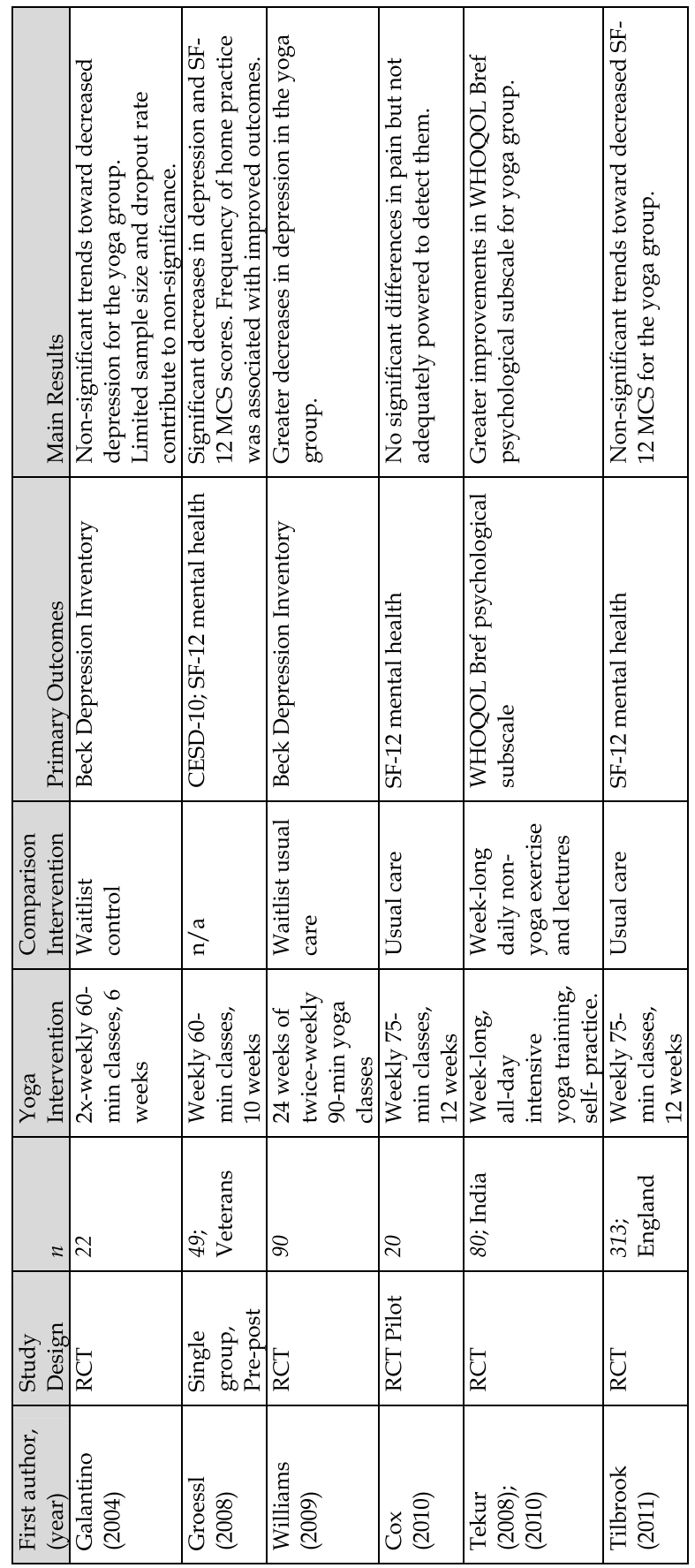

Table 4. Studies that examined the effect of yoga on psychological health. 
Overall, our results are similar to and confirmatory of those published in a review article in 2011. This prior review was useful, but was released before the results of the two largest randomized controlled trials that have been published to date. Thus, we believed it was important to add these studies to the body of existing literature. We conclude that yoga interventions impact multiple outcomes that are important to the health and well-being of people afflicted with chronic low back pain. Recent, high quality evidence suggests that yoga provides comparable effects to, and is more appealing than, formal stretching and strengthening programs led by physical therapists. The recent data suggest that it would behoove health care organizations and the members they serve, to have yoga as an available health service option at little or no cost.

\section{References}

Anderson, G. B. J. "The Epidemiology of Spinal Disorders." In The Adult Spine: Principles and Practice., edited by JW Frymoyer, 93-141. New York: Raven Press, 1997.

Barnes, P. M., E. Powell-Griner, K. McFann, and R. L. Nahin. "Complementary and Alternative Medicine Use among Adults: United States, 2002." Adv Data, no. 343 (2004): 1-19.

Barnes, P. M., B. Bloom, and R. L. Nahin. "Complementary and Alternative Medicine Use among Adults and Children: United States, 2007." Natl Health Stat Report 10, no. 12 (2009): 1-23.

Broad, W. J. "How Yoga Can Wreck Your Body." New York Times, January 5, 20122012.

Choo, P. W., C. S. Rand, T. S. Inui, M. L. Lee, E. Cain, M. Cordeiro-Breault, C. Canning, and R. Platt. "Validation of Patient Reports, Automated Pharmacy Records, and Pill Counts with Electronic Monitoring of Adherence to Antihypertensive Therapy." Med Care 37, no. 9 (1999): 846-57.

Chou, R., A. Qaseem, V. Snow, D. Casey, J. T. Cross, Jr., P. Shekelle, and D. K. Owens. "Diagnosis and Treatment of Low Back Pain: A Joint Clinical Practice Guideline from the American College of Physicians and the American Pain Society." Ann Intern Med 147, no. 7 (2007): 478-91.

Chou, R., and L. H. Huffman. "Medications for Acute and Chronic Low Back Pain: A Review of the Evidence for an American Pain Society/American College of Physicians Clinical Practice Guideline." Ann Intern Med 147, no. 7 (2007): 505-14.

Chou, R., and L. H. Huffman . "Nonpharmacologic Therapies for Acute and Chronic Low Back Pain: A Review of the Evidence for an Aps/American College of Physicians Clinical Practice Guideline." Ann Intern Med 147, no. 7 (2007): 492-504.

Cox, H., H. Tilbrook, J. Aplin, A. Semlyen, D. Torgerson, A. Trewhela, and I. Watt. "A Randomised Controlled Trial of Yoga for the Treatment of Chronic Low Back Pain: Results of a Pilot Study." Complement Ther Clin Pract 16, no. 4 (2010): 187-93.

Currie, S. R., and J. Wang. "Chronic Back Pain and Major Depression in the General Canadian Population." Pain 107, no. 1-2 (2004): 54-60.

Deyo, R. A., S. K. Mirza, and B. I. Martin. "Back Pain Prevalence and Visit Rates: Estimates from U.S. National Surveys, 2002." Spine 31, no. 23 (2006): 2724-7.

Evans, D. D., M. Carter, R. Panico, L. Kimble, J. T. Morlock, and M. J. Spears. "Characteristics and Predictors of Short-Term Outcomes in Individuals Self-Selecting Yoga or Physical Therapy for Treatment of Chronic Low Back Pain." Pm R 2, no.11 (2010): 1006-15.

Galantino, M. L., T. M. Bzdewka, J. L. Eissler-Russo, M. L. Holbrook, E. P. Mogck, P. Geigle, and J. T. Farrar. "The Impact of Modified Hatha Yoga on Chronic Low Back Pain: A Pilot Study." Altern Ther Health Med 10, no. 2 (2004): 56-9. 
Groessl, E. J., T. G. Ganiats, and A. J. Sarkin. "Sociodemographic Differences in Quality of Life in Rheumatoid Arthritis." Pharmacoeconomics 24, no. 2 (2006): 109-21.

Groessl, E. J., K. R. Weingart, K. Aschbacher, L. Pada, and S. Baxi. "Yoga for Veterans with Chronic Low-Back Pain." J Altern Complement Med 14, no. 9 (2008): 1123-29.

Groessl, E.J., K. R. Weingart, N. Johnson, and S. Baxi. "The Benefits of Yoga for Women Veterans with Chronic Low Back Pain." J Altern Complement Med (in press).

Johnson, Andrew, and Richards Marged. "Celebrity Power Yoga: The New Craze from over There Causing Bad Karma over Here." The Independent. (U. K.) , Sunday, 30 January 2005.

Liu, H., C. E. Golin, L. G. Miller, R. D. Hays, C. K. Beck, S. Sanandaji, J. Christian, T. Maldonado, D. Duran, A. H. Kaplan, and N. S. Wenger. "A Comparison Study of Multiple Measures of Adherence to Hiv Protease Inhibitors." Ann Intern Med 134, no. 10 (2001): 968-77.

Luo, X., R. Pietrobon, S. X. Sun, G. G. Liu, and L. Hey. "Estimates and Patterns of Direct Health Care Expenditures among Individuals with Back Pain in the United States." Spine 29, no. 1 (2004): 79-86.

Posadzki, P., and E. Ernst. "Yoga for Low Back Pain: A Systematic Review of Randomized Clinical Trials." Clin Rheumatol 30, no. 9 (2011): 1257-62.

Saper, R. B., K. J. Sherman, D. Cullum-Dugan, R. B. Davis, R. S. Phillips, and L. Culpepper. "Yoga for Chronic Low Back Pain in a Predominantly Minority Population: A Pilot Randomized Controlled Trial." Altern Ther Health Med 15, no. 6 (2009): 18-27.

Sherman, K. J., D. C. Cherkin, J. Erro, D. L. Miglioretti, and R. A. Deyo. "Comparing Yoga, Exercise, and a Self-Care Book for Chronic Low Back Pain: A Randomized, Controlled Trial." Ann Intern Med 143, no. 12 (2005): 849-56.

Sherman, K. J., D. C. Cherkin, R. D. Wellman, A. J. Cook, R. J. Hawkes, K. Delaney, and R. A. Deyo. "A Randomized Trial Comparing Yoga, Stretching, and a Self-Care Book for Chronic Low Back Pain." Arch Intern Med (2011).

Sullivan, M. J., K. Reesor, S. Mikail, and R. Fisher. "The Treatment of Depression in Chronic Low Back Pain: Review and Recommendations." Pain 50, no. 1 (1992): 5-13.

Tekur, P., S. Chametcha, R. N. Hongasandra, and N. Raghuram. "Effect of Yoga on Quality of Life of Clbp Patients: A Randomized Control Study." Int J Yoga 3, no. 1 (2010): 10-7.

Tekur, P., C. Singphow, H. R. Nagendra, and N. Raghuram. "Effect of Short-Term Intensive Yoga Program on Pain, Functional Disability and Spinal Flexibility in Chronic Low Back Pain: A Randomized Control Study." J Altern Complement Med 14, no. 6 (2008): 637-44.

Telles, S., M. Dash, and K. V. Naveen. "Effect of Yoga on Musculoskeletal Discomfort and Motor Functions in Professional Computer Users." Work 33, no. 3 (2009): 297-306.

Tilbrook, H. E., H. Cox, C. E. Hewitt, A. R. Kang'ombe, L. H. Chuang, S. Jayakody, J. D. Aplin, A. Semlyen, A. Trewhela, I. Watt, and D. J. Torgerson. "Yoga for Chronic Low Back Pain: A Randomized Trial." Ann Intern Med 155, no. 9 (2011): 569-78.

Ülger, Ö., and N. V. Yağli. "Effects of Yoga on Balance and Gait Properties in Women with Musculoskeletal Problems: A Pilot Study." Complement Ther Clin Pract 17, no. 1 (2011): 13-5.

Williams, K. A., J. Petronis, D. Smith, D. Goodrich, J. Wu, N. Ravi, E. J. Doyle, Jr., R. Gregory Juckett, M. Munoz Kolar, R. Gross, and L. Steinberg. "Effect of Iyengar Yoga Therapy for Chronic Low Back Pain." Pain 115, no. 1-2 (2005): 107-17.

Williams, K., C. Abildso, L. Steinberg, E. Doyle, B. Epstein, D. Smith, G. Hobbs, R. Gross, G. Kelley, and L. Cooper. "Evaluation of the Effectiveness and Efficacy of Iyengar Yoga Therapy on Chronic Low Back Pain." Spine (Phila Pa 1976) 34, no. 19 (2009): 2066-76. 


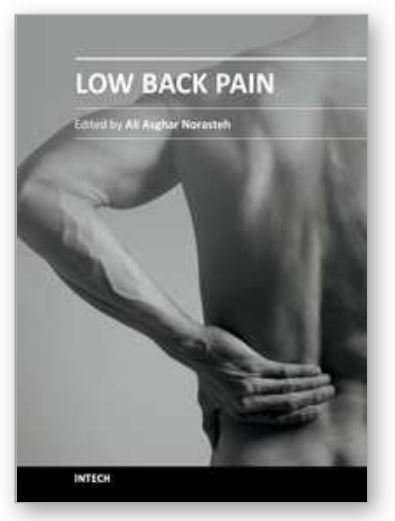

\section{Low Back Pain}

Edited by Dr. Ali Asghar Norasteh

ISBN 978-953-51-0599-2

Hard cover, 352 pages

Publisher InTech

Published online 09, May, 2012

Published in print edition May, 2012

This book includes two sections. Section one is about basic science, epidemiology, risk factors and evaluation, section two is about clinical science especially different approach in exercise therapy. I envisage that this book will provide helpful information and guidance for all those practitioners involved with managing people with back pain-physiotherapists, osteopaths, chiropractors and doctors of orthopedics, rheumatology, rehabilitation and manual medicine. Likewise for students of movement and those who are involved in re-educating movement-exercise physiologists, Pilates and yoga teachers etc.

\section{How to reference}

In order to correctly reference this scholarly work, feel free to copy and paste the following:

Erik J. Groessl, Marisa Sklar and Douglas Chang (2012). Yoga as a Treatment for Low Back Pain: A Review of the Literature", Low Back Pain, Dr. Ali Asghar Norasteh (Ed.), ISBN: 978-953-51-0599-2, InTech, Available from: http://www.intechopen.com/books/low-back-pain/yoga-as-a-treatment-for-low-back-pain-a-review-of-theliterature

\section{INTECH}

open science | open minds

\section{InTech Europe}

University Campus STeP Ri

Slavka Krautzeka 83/A

51000 Rijeka, Croatia

Phone: +385 (51) 770447

Fax: +385 (51) 686166

www.intechopen.com

\section{InTech China}

Unit 405, Office Block, Hotel Equatorial Shanghai

No.65, Yan An Road (West), Shanghai, 200040, China

中国上海市延安西路65号上海国际贵都大饭店办公楼 405 单元

Phone: +86-21-62489820

Fax: $+86-21-62489821$ 
(C) 2012 The Author(s). Licensee IntechOpen. This is an open access article distributed under the terms of the Creative Commons Attribution 3.0 License, which permits unrestricted use, distribution, and reproduction in any medium, provided the original work is properly cited. 